\title{
Favorable outcome of repeat mechanical thrombectomy in a geriatric patient: illustrative case
}

\author{
Ali A. Alsarah, MD, ${ }^{1}$ Omar M. Hussein, MD, ${ }^{1}$ and Andrew P. Carlson, MD, MD-CR ${ }^{2}$ \\ Departments of ${ }^{1}$ Neurology and ${ }^{2}$ Neurosurgery, University of New Mexico Hospital, Albuquerque, New Mexico
}

BACKGROUND The authors presented their experience with a case of repeat thrombectomy in a 93-year-old patient who showed a favorable outcome after recurrent large vessel occlusion treated with emergency mechanical thrombectomy.

OBSERVATIONS Mechanical thrombectomy has been proven to be effective in treating large vessel occlusion types of ischemic stroke. Most of the patient populations involved in the thrombectomy-related studies were younger than 80 years. In addition, recurrent mechanical thrombectomy is not a common procedure in clinical practice. This unusual case demonstrated the potential to achieve a favorable outcome with thrombectomy even in a patient older than 85 years with recurrent large vessel occlusion.

LESSONS There can be a favorable neurological outcome after one or repeat thrombectomies for geriatric patients older than 90 years, and age should not be a deterrent to treatment.

https://thejns.org/doi/abs/10.3171/CASE2140

KEYWORDS ischemic stroke; mechanical thrombectomy; geriatric patients; large vessel occlusion

From 2015 to 2018, seven trials (MR CLEAN, ${ }^{1}$ REVA SCAT, ${ }^{2}$ ESCAPE, ${ }^{3}$ SWIFT PRIME, ${ }^{4}$ EXTEND-1A, ${ }^{5}$ DIFFUSE $3,{ }^{6}$ and $\mathrm{DAWN}^{7}$ ) provided evidence of increased functional independence without increasing the risk of death after the early performance of mechanical thrombectomy. Intravenous chemical thrombolysis and mechanical thrombectomy have become the gold standards for treating patients who experience acute ischemic stroke. ${ }^{8}$

Despite its rarity, recurrent mechanical thrombectomy has been considered a relatively safe procedure. Bouslama et al. ${ }^{9}$ found no statistically significant differences in demographics, stroke severity, time from last known normal to puncture, reperfusion rates, hemorrhagic complications, good clinical outcomes, and mortality between patients who underwent repeat thrombectomy and patients who had a single thrombectomy. That finding reflected a favorable neurological outcome. ${ }^{9}$ The role of mechanical thrombectomy in patients older than 85 years has also been debated, given the overall risk of a worse outcome in this population. Recent studies, however, have shown that a patient population older than 85 years may still benefit from thrombectomy, especially with complete recanalization, despite the risk. ${ }^{10}$ We present a case study of a 93-year-old woman who showed significant improvement in functional outcome after each of the two thrombectomies she underwent.

\section{Illustrative Case}

A 93-year-old woman with a past medical history of hypertension, hyperlipidemia, and gout presented with the acute onset of left-sided weakness and left-sided facial droop. She was last seen well at 1:00 PM, and her National Institutes of Health Stroke Scale (NIHSS) score upon arrival was 22. The patient received alteplase at 2:21 PM. A right middle cerebral artery (MCA) M1 occlusion was revealed on computed tomography angiography (CTA) and diagnostic cerebral angiography (DCA) (Fig. 1A). CTA showed mild calcified atherosclerotic plaques at the carotid bifurcation bilaterally but no significant intracranial atherosclerosis.

The patient underwent successful right M1 mechanical thrombectomy and had a Thrombolysis in Cerebral Infarction (TICl) score of 3 at 4:15 PM (Fig. 1B). Her premorbid modified Rankin Scale (mRS) score was 0 . Magnetic resonance imaging (MRI) of the brain showed

ABBREVIATIONS CT = computed tomography; CTA = CT angiography; $\mathrm{DCA}=$ diagnostic cerebral angiography; $\mathrm{MCA}=$ middle cerebral artery; MRI = magnetic resonance imaging; $\mathrm{mRS}=$ modified Rankin Scale; NIHSS = National Institutes of Health Stroke Scale; TICl = Thrombolysis in Cerebral Infarction.

INCLUDE WHEN CITING Published June 14, 2021; DOI: 10.3171/CASE2140.

SUBMITTED January 22, 2021. ACCEPTED February 11, 2021.

(C) 2021 The authors, CC BY-NC-ND 4.0 (http://creativecommons.org/licenses/by-nc-nd/4.0/). 

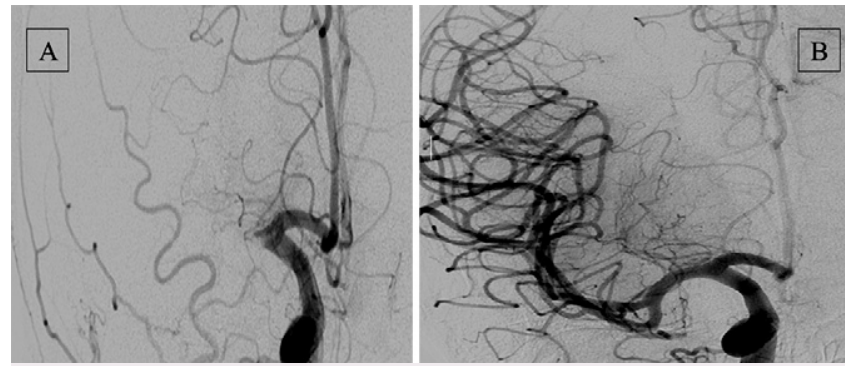

FIG. 1. DCA. A: Acute right MCA M1 occlusion. B: After mechanical thrombectomy revascularization of the entire MCA territory with no evidence of distal thromboembolic complication or persistent occlusion. restriction diffusion of the right basal ganglia without evidence of hemorrhage. Her discharge NIHSS score was 0 . The etiology of the stroke was concluded to be cryptogenic after thorough stroke workup, including transthoracic echocardiography with bubble study, thyroid function test, and lipid profile, which did not reveal significant abnormality. She was discharged with instructions to take aspirin $81 \mathrm{mg}$ and atorvastatin $80 \mathrm{mg}$ daily.

Fifty-two days later, at 10:27 AM, the patient presented with the acute onset of left-sided weakness and slurred speech. Her NIHSS score upon arrival was 8. The patient was last seen well at 3:00 AM and thus was not a candidate for chemical thrombolysis. Head computed tomography (CT) showed no acute infarct. CTA showed occlusion of the right proximal M2 superior division of the MCA. CT perfusion of the brain showed ischemic penumbra of $55 \mathrm{~mL}$ without an ischemic core in the respective vascular territory (Fig. 2A). She underwent mechanical thrombectomy at 12:10 PM with $\mathrm{TICl} 3$ revascularization (Fig. 3). MRI showed small-to-moderate volume, right MCA territorial ischemic infarct with minimal petechial hemorrhage (Fig. 2B). Her NIHSS score at discharge was 0 . Her collateral circulation assessment based on the CTA was overall good and was considered to have
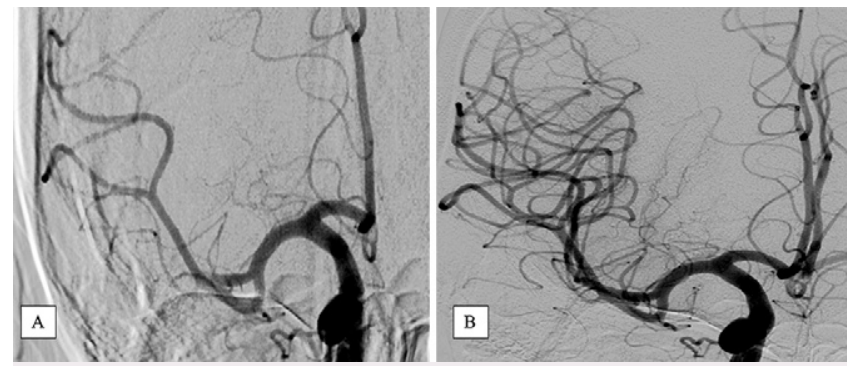

FIG. 3. Second DCA showing (A) acute right MCA M2 occlusion and (B) post-mechanical thrombectomy revascularization of the entire MCA territory with no evidence of distal thromboembolic complication or persistent occlusion.

the following scores according to single-phase CTA collateral scores in acute stroke: Tan et al. $=2$; Maas et al. $=2 / 3$; Souza et al. $=2 / 3$; Cristoforidis $=2$; Miteff $=2$; and ASPECTS $=$ approximately $3 .{ }^{11-18}$

After her second stroke, a cardiac loop recorder was implanted. It did not reveal any evidence of dysrhythmia, which confirmed the diagnosis of cryptogenic stroke. The patient was started on apixaban $2.5 \mathrm{mg}$.

\section{Discussion}

\section{Observations}

The HERMES meta-analysis favored endovascular thrombectomy even in patient populations older than 80 years, noting that it significantly reduced disability at 90 days as compared to that in the standard medical treatment arm of the study. ${ }^{19}$ This meta-analysis included 198 patients with an age $\geq 80$ years, and the rate of an mRS score of 0 to 2 at 90 days was $29.8 \%$ in the endovascular group and $13.9 \%$ in the control group (OR 3.68, 95\% Cl 1.95-6.92). However, the rate of an mRS score of 0 to 2 at 90 days in the endovascular group decreased with advancing age: 50 to 59 years, $50.0 \%$; 60 to 69 years, $51.9 \% ; 70$ to 79 years, $43.1 \%$; and $\geq 80$ years, $29.8 \%$. Therefore, this meta-analysis
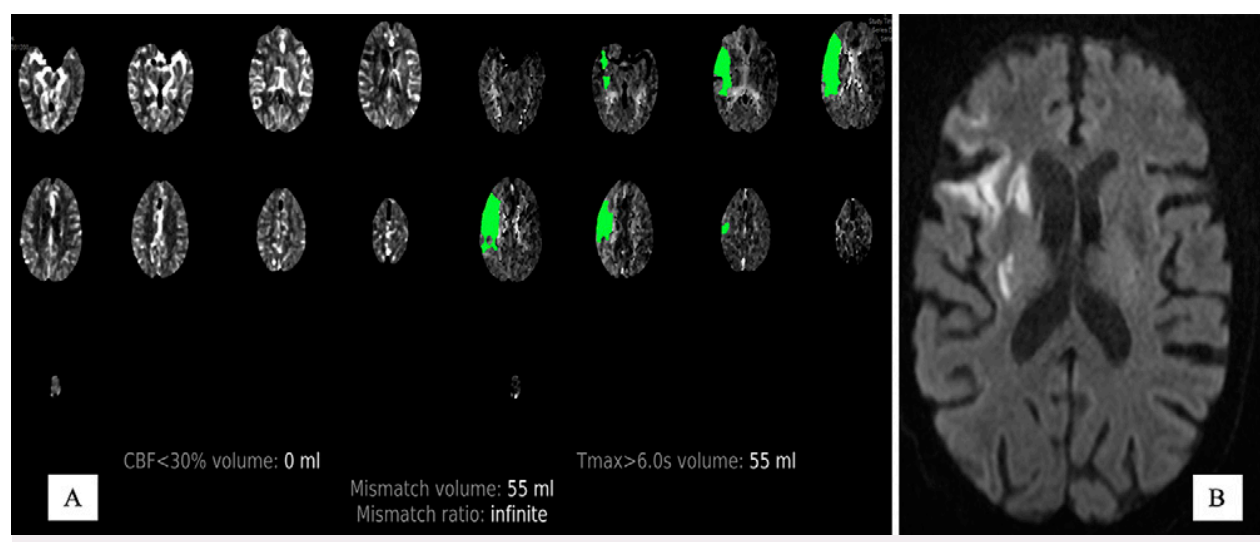

FIG. 2. A: CT perfusion scans of head with contrast showing increase in time to maximum contrast intensity on the ct perfusion (Tmax) $>6$ seconds in the right superior MCA territory. There is no decreased cerebral blood flow $<30 \%$. B: Diffusion-weighted MRI, axial section, showing restricted diffusion in the right MCA territory notably involving right anterolateral frontal lobe, insula, and basal ganglia. 
suggested that age remained a strong independent negative predictor of outcome despite the treatment effect of mechanical thrombectomy.

Imahori et al. demonstrated significant differences in procedure times for endovascular treatment between patients whose age was $\geq 80$ years and those whose age was $<80 .^{18}$ They found that being 80 years or older was not a significant predictor for outcomes after mechanical thrombectomy ( $42 \%$ vs $57 \%$, respectively; $p=0.261$ ) or death ( $8 \%$ vs $5 \%$, respectively; $p=0.653$ ), whereas complete recanalization was an influential predictor with a positive outcome, particularly in those 80 years or older with complete recanalization $(\mathrm{mTICl} 3) .^{18}$

Recurrent mechanical thrombectomy is generally not contraindicated; in fact, it appears safe in properly selected patients with recurrent large vessel occlusion strokes. Bouslama et al. ${ }^{9}$ studied a group of patients who underwent repeat thrombectomy. Although none of the selected patients was more than 90 years old, they showed favorable outcomes without a significant increased risk of intracranial hemorrhage or significant increase in the risk of death. ${ }^{9}$ Six of the 15 patients involved in the study had ipsilateral (same stream) repeat thrombectomies. At 90 days, the mRS score for 4 of the 6 did not show worsening, 1 showed improvement, and 1 had a worse $\mathrm{mRS}$ score after a third stroke. A recent study by Lee et al. also concluded that ipsilateral repeat thrombectomies produced a significantly better clinical outcome when compared to that with contralateral repeat thrombectomies $(83.3 \%$ vs $16.7, p=0.027),{ }^{19}$ but none of the patients was older than 90 years.

\section{Lessons}

Repeat mechanical thrombectomy is an uncommon procedure in clinical practice, and to our knowledge, this is the first reported patient older than 90 years with a good functional outcome after both interventions. Although the overall outcomes in such patient populations may be worse than in younger populations and first-time thrombectomy patients, the current literature supports an aggressive approach even in these groups. The limitations of this approach for geriatric patients are multifactorial, including stroke size, medical comorbidities, operative and postoperative complications, and adverse effects of the secondary stroke prevention measures, which can further challenge the outcome. Further retrospective studies are necessary to analyze the benefits and challenges of performing thrombectomies in patients older than 90 years to build on the literature and find potential positive outcomes. Moreover, it is necessary to consider that as the aging population increases, more dynamic studies will be necessary to tend to these individuals.

\section{References}

1. Berkhemer OA, Fransen PS, Beumer D, et al. A randomized trial of intraarterial treatment for acute ischemic stroke. $N$ Engl $\mathrm{J}$ Med. 2015;372(1):11-20.

2. Jovin TG, Chamorro A, Cobo E, et al. Thrombectomy within 8 hours after symptom onset in ischemic stroke. N Engl J Med. 2015;372(24): 2296-2306.

3. Goyal M, Demchuk AM, Menon BK, et al. Randomized assessment of rapid endovascular treatment of ischemic stroke. N Engl J Med. 2015;372(11):1019-1030.

4. Saver JL, Goyal M, Bonafe A, et al. Stent-retriever thrombectomy after intravenous t-PA vs. t-PA alone in stroke. N Engl J Med. 2015; 372(24):2285-2295.

5. Campbell BC, Mitchell PJ, Kleinig TJ, et al. Endovascular therapy for ischemic stroke with perfusion-imaging selection. N Engl J Med. 2015;372(11):1009-1018.
6. Albers GW, Lansberg MG, Kemp S, et al. A Multicenter Randomized Controlled Trial of Endovascular Therapy Following Imaging Evaluation for Ischemic Stroke (DEFUSE 3). SAGE Publications; 2017.

7. Nogueira RG, Jadhav AP, Haussen DC, et al. Thrombectomy 6 to 24 hours after stroke with a mismatch between deficit and infarct. $N$ Engl J Med. 2018;378(1):11-21.

8. Goyal M, Menon BK, van Zwam WH, et al. Endovascular thrombectomy after large-vessel ischaemic stroke: a meta-analysis of individual patient data from five randomised trials. Lancet. 2016;387 (10029):1723-1731.

9. Bouslama M, Haussen DC, Rebello LC, et al. Repeated mechanical thrombectomy in recurrent large vessel occlusion acute ischemic stroke. Intervent Neurol. 2017;6(1-2):1-7.

10. Seker F, Potreck A, Möhlenbruch M, et al. Comparison of four different collateral scores in acute ischemic stroke by $\mathrm{CT}$ angiography. J Neurointerv Surg. 2016;8(11):1116-1118.

11. Tan IYL, Demchuk AM, Hopyan J, et al. CT angiography clot burden score and collateral score: correlation with clinical and radiologic outcomes in acute middle cerebral artery infarct. $A m \mathrm{~J}$ Neuroradiol. 2009;30(3):525-531.

12. Maas MB, Lev MH, Ay H, et al. Collateral vessels on CT angiography predict outcome in acute ischemic stroke. Stroke. 2009;40(9): 3001-3005

13. Souza LCS, Yoo AJ, Chaudhry ZA, et al. Malignant CTA collateral profile is highly specific for large admission DWI infarct core and poor outcome in acute stroke. Am J Neuroradiol. 2012;33(7): 1331-1336.

14. Christoforidis GA, Mohammad Y, Kehagias D, et al. Angiographic assessment of pial collaterals as a prognostic indicator following intra-arterial thrombolysis for acute ischemic stroke. Am J Neuroradiol. 2005;26(7):1789-1797.

15. Miteff F, Levi CR, Bateman GA, et al. The independent predictive utility of computed tomography angiographic collateral status in acute ischaemic stroke. Brain. 2009;132(Pt 8):2231-2238.

16. Barber PA, Demchuk AM, Zhang J, Buchan AM. Validity and reliability of a quantitative computed tomography score in predicting outcome of hyperacute stroke before thrombolytic therapy. Lancet. 2000;355(9216):1670-1674.

17. Seker F, Potreck A, Möhlenbruch M, et al. Comparison of four different collateral scores in acute ischemic stroke by CT angiography. J Neurointerv Surg. 2016;8(11):1116-1118.

18. Imahori T, Tanaka K, Arai A, et al. Mechanical thrombectomy for acute ischemic stroke patients aged 80 years or older. J Stroke Cerebrovasc Dis. 2017;26(12):2793-2799.

19. Lee HJ, Kwak HS, Chung GH, Park JS. Repeated endovascular thrombectomy in patients with acute ischemic stroke in a single center. J Stroke Cerebrovasc Dis. 2021;30(1):105457.

\section{Disclosures}

Dr. Carlson reported other support from Cerebroscope outside the submitted work.

\section{Author Contributions}

Conception and design: all authors. Acquisition of data: Alsarah, Hussein. Analysis and interpretation of data: Alsarah, Hussein. Drafting the article: Carlson, Alsarah. Critically revising the article: Carlson, Hussein. Reviewed submitted version of manuscript: all authors. Approved the final version of the manuscript on behalf of all authors: Carlson. Study supervision: Hussein.

\section{Correspondence}

Andrew P. Carlson: University of New Mexico, Albuquerque, NM. andrewcarlson@salud.unm.edu. 